(c) 2002 IPEM. Published by Elsevier Science Ltd. All rights reserved. Used with permission. This paper was originally published in the Elsevier Science Journal, Medical Engineering \& Physics. Vol. 24, Issue 2, March 2002. p.129-138. Available at:

http://www.elsevier.com/locate/issn/13504533

Keywords: Lacunarity; Texture; Bone quality; Trabecular bone; Computed tomography

\title{
Lacunarity analysis of spatial pattern in CT images of vertebral trabecular bone for assessing osteoporosis
} Geoffrey Dougherty a, Geoffrey M. Henebry b

a Department of Radiologic Sciences, Faculty of Allied Health Sciences, Kuwait University, P.O. Box 31470, 90805 Sulaibikhat, Kuwait

๖ Center for Advanced Land Management Information Technologies, University of Nebraska, Lincoln, NE 68588-0517, USA

\begin{abstract}
The structural integrity of vertebral trabecular bone is determined by the continuity of its trabecular network and the size of the holes comprising its marrow space, both of which determine the apparent size of the marrow spaces in a transaxial CT image. A modelindependent assessment of the trabeculation pattern was determined from the lacunarity of thresholded CT images. Using test images of lumbar vertebrae from human cadavers, acquired at different slice thicknesses, we determined that both median thresholding and local adaptive thresholding (using a $7 \cdot 7$ window) successfully segmented the grey-scale images. Lacunarity analysis indicated a multifractal nature to the images, and a range of marrow space sizes with significant structure around $14-18 \mathrm{~mm}^{2}$. Preliminary studies of in vivo images from a clinical CT scanner indicate that lacunarity analysis can follow the pattern of bone loss in osteoporosis by monitoring the homogeneity of the marrow spaces, which is related to the connectivity of the trabecular bone network and the marrow space sizes. Although the patient sample was small, derived parameters such as the maximum deviation of the lacunarity from a neutral (fractal) model, and the maximum derivative of this deviation, seem to be sufficiently sensitive to distinguish a range of bone conditions. Our results suggest that these parameters, used with bone mineral density values, may have diagnostic value in characterizing osteoporosis and predicting fracture risk.
\end{abstract}

\section{Introduction}

Osteoporosis is a complex, multifactorial metabolic disorder characterized by a loss of bone strength due to diminished bone mass and deterioration of the microarchitecture of trabecular and cortical bone. Whilst the most common method of assessing bone strength is to monitor loss of bone mass by bone mineral densitometry, the role of trabecular bone structure has increasingly been recognized as a significant contributory factor [1,2]. Quantitative computed tomography (QCT) is able to isolate and image trabecular bone in vivo, but commercial clinical CT scanners have limited spatial resolution $(\sim 0.5 \mathrm{~mm})$ and are unable to properly resolve vertebral trabeculae. Modified peripheral CT systems $[3,4]$ 
with improved spatial resolution ( $\sim 0.20 \mathrm{~mm}$ ), experimental high resolution (100-150 $\mu \mathrm{m})$ scanners [5] and micro-computed tomography with spatial resolutions of around 15 $\mu \mathrm{m}$ [6] have all shown trabecular structure in the radius and the tibia. However, the high dose required to obtain images of the central skeleton at this resolution could not be justified for in vivo diagnosis of osteoporosis. Despite their resolution limitations clinical two dimensional axial images of thin vertebral slices will contain some architectural information, albeit in a blurred form which we will refer to as texture. The challenge is to identify textural parameters that can be determined in vivo and which provide sufficient structural information that they add additional sensitivity to the bone mineral density (BMD) values commonly used as an indicator of osteoporosis.

One of the simplest approaches for describing texture is to use moments of the greylevel histogram (e.g. pixel mean and variance) of the image. However, this analysis is necessarily limited since the whole image is considered to be the tonal primitive and is characterized statistically: information on spatial organization or periodicity is lost $[7,8]$. We have previously reported that firstorder measures are not a useful indicator of texture in CT images of trabecular bone, but that second-order measures such as fractal dimension and lacunarity are able to characterize the texture of such images $[9,10]$. Lacunarity was originally developed to describe a property of fractals [11,12], but is not predicated on selfsimilarity (i.e. fractalness) and can be used to describe the spatial distribution of real data sets [13]. This is a distinct advantage over fractal dimension, which has been commonly used as a compact descriptor of texture in medical images that often exhibit little self-similarity. Lacunarity plots explicitly characterize the spatial organization of an image, including the average size of any structural sub-unit(s) within an image, making them potentially useful in representing the trabecular thinning and perforation of vertebral trabecular bone associated with osteoporosis. The lacunarity algorithm is simple to implement, depending only on local means and variances calculated for different window sizes throughout the image. It is a mass-oriented metric that is insensitive to image boundaries, which complicate analysis by discrete Fourier transform. Lacunarity plots are robust to the presence of noise and blurring within the imaging system so that corrections for these effects need not be implemented for most studies. They can be generated in near real-time, following image acquisition and binarization.

Lacunarity analysis has been successfully used in landscape ecology with optical [14] and synthetic aperture radar [15] images. Grey-scale images were pre-processed to derive an ordered series of binary images based on quartiles of the image histogram which were then analysed separately. This treatment was successful because each binary image could be associated with scene features related to interactions with the illuminating radiation. However, the preprocessing by quantiles is arbitrary and is likely to introduce some bias; in most medical imaging the objects of interest are well-defined and known a priori, so that a single thresholding to produce two classes (foreground and background) is likely to be more appropriate.

There are numerous thresholding schemes that can be used to segment grey-scale images and the selection of a particular method is often governed by the level of noise present and the image contrast [16]. Analysis of the resulting binarized images can produce significant differences. We compared the use of three global thresholding 
schemes (using the mean and median density of the images, and a method based on discriminant analysis [17]) and a local adaptive thresholding method using a 7.7 window [18] on a set of 16 test images of vertebral bone in order to assess the most appropriate scheme for such images. We investigated the limits of sensitivity of the lacunarity technique in delivering architectural information, such as the degree of heterogeneity within the image and the size of any structural sub-unit. The technique was then applied to a set of ten clinical images, taken from female volunteers with a range of bone densities, to determine its potential in distinguishing osteoporotic from non-osteoporotic patients.

\section{Materials and methods}

\subsection{Test images}

The lumbar vertebrae (L4) from four female human donors (mean age 45 \pm 2 years) were collected at autopsy. All had died suddenly from accidents, and none had a history of metabolic bone disease or fractures. The vertebrae were soaked in a dilute formalin solution for one week to rehydrate the bone and eliminate trapped air from the trabeculae. Axial CT images of the vertebrae were obtained using a previously developed protocol [10], and a scan field of view (FOV) of $25 \mathrm{~cm}$. Scans were taken through the middle of each vertebra, parallel to the vertebral endplates, using slice thicknesses of 1,3,5 and 10 $\mathrm{mm}$ and a scan time of 3s. Each image was stored as 512.512 pixels with 12-bit quantization, although it was convenient for further analysis to rescale the pixel values to the more familiar range of $[0,255]$. A standard mineral-equivalent bone phantom (QCT phantom, Image Analysis Inc., Irvine, CA) was included in each view to calibrate the pixel values so that the average BMDs could be obtained.

For each of the 16 images, a square (48.48 pixels) region of interest (ROI) was extracted close to the anterior surface of the vertebra and comprising mostly trabecular bone. We have previously shown that selecting adjacent ROIs up to 4 pixels apart does not significantly affect the resulting lacunarity estimates [10]. Each group of ROIs from a particular vertebra was spatially registered by translating and rotating the four ROIs into alignment based on three fiducial points chosen from each. Identically registered ROIs of $32 \cdot 32$ pixels, comprising trabecular bone only, were then extracted for subsequent analysis as test images.

\subsection{Clinical images}

Images of the L4 vertebrae of ten female Kuwaiti volunteers (mean age 48.8 \pm 3.3 years), labelled A-J (Table 1), were taken in vivo using the same technique factors as for the test images, but with a scan FOV of $35 \mathrm{~cm}$ (to include larger patients) and a slice thickness of $5 \mathrm{~mm}$ (to enable BMD and texture estimates to be made from the same image). These patients had been referred to the Faculty of Allied Health Sciences as part of their clinical evaluation of osteoporosis. None of the subjects had a history of bone fracture or major systemic disorder: all gave informed consent. The study was approved by the Ethics Committee of the Health Sciences Center, Kuwait University. A ROI of 
32.32 pixels, comprising trabecular bone close to the anterior surface of the vertebra, was directly extracted from each of these CT images and these are henceforth referred to as the clinical images. Spatial registration was not appropriate.

Table 1. Measured BMDs and corresponding designations $\mathrm{a}_{\mathrm{a}}$

\begin{tabular}{llll}
\hline Patient & Age (years) & $\mathrm{BMD}\left(\mathrm{mg} \mathrm{cm}^{-3}\right)$ & Designation \\
\hline A & 49 & 204.0 & High \\
B & 45 & 205.0 & High \\
C & 47 & 165.0 & Normal \\
D & 50 & 150.0 & Normal \\
E & 52 & 136.0 & Normal \\
F & 47 & 128.0 & Low \\
G & 53 & 98.0 & Low \\
H & 54 & 82.0 & Very low \\
I & 46 & 103.0 & Very low \\
J & 45 & 102.5 & Very low \\
\hline
\end{tabular}

a Compared to age- and sex-matched normatives [20], the BMDs are considered normal if they are within \pm 1 standard deviation (SD) from the normative, low/high if they are 1-2 SDs lower/higher than the normative, and very low/very high if they are more than 2 SDs below/above the normative.

\subsection{Summary statistics}

The compression rate and entropy of an image characterize the complexity of the image and may indicate the degree of texture therein. The compression rates and entropies were obtained using MKS Toolkit 6.1 (Mortice Kern Systms Inc., Waterloo, Canada). The effective (lossless) compression rates relate to the expressed bandwidth (grey-scale range) for Huffman compression. The entropy of an image is a measure of its information content. Low compression rates and high entropy values indicate a more complicated image, with little redundancy and a lot of randomness (and, hence, a higher fractal dimension).

\subsection{Fractal dimension}

The fractal dimension of each image, taken as an average over the mid-range of spatial frequencies, was computed using the Fourier power spectrum algorithm [19]. The effect of blurring by the CT scanner was removed by dividing the radial power spectra of the images by the square of the scanner MTF [10] resulting in estimates of fractal dimension within the theoretically allowed range 2.0-3.0.

\subsection{Lacunarity analysis}

Lacunarity, $L(\mathrm{r})$, can be defined in terms of the local first and second moments (i.e., local mean and variance) measured for different neighbourhood sizes, $r$, about every pixel in an image [13], i.e.

$L(\mathrm{r})=1+\left\{\operatorname{var}(\mathrm{r}) / \operatorname{mean}^{2}(\mathrm{r})\right\}$

where mean(r) and var(r) are the mean and variance, respectively, for a neighbourhood size, $r$, using all neighbourhoods of that size within the image (viz. exhaustive sampling). 
The lacunarity was measured from the binarized images as is common practice $[13,15]$. It is in effect a mean normalized variance, calculated as a function of neighbourhood size. The lacunarity as a function of neighbourhood size is generally presented as a log-log plot, which illustrates the scale dependency of spatial nonstationarity in the image. The use of a square neighbourhood constrains the manifestation of the spatial heterogeneity to translational invariance. Higher lacunarity values indicate more translational invariance (higher contagion), i.e. a wider range of sizes of structures within an image. The decay pattern of the lacunarity plot contains significant information about the spatial structure of the image $[10,15]$. The lacunarity plot for a selfsimilar fractal, for example, would be linear.

In the case of lacunarity plots that are close to linear, it is instructive to plot their deviation from a straight line representing a self-similar fractal (viz., no change in spatial pattern or texture with window size). This will emphasize subtle differences that are not conspicuous in the decay curves themselves, and is useful in identifying size ranges for different tonal features. Positive (negative) deviations indicate greater (less) spatial homogeneity than the scale-invariant (fractal) neutral model, and a prominent maximum (obtained from the derivative of the deviations) will indicate the typical size of a structuring element (e.g., marrow space) in the image.

\section{Results}

\subsection{General observations}

The mean BMDs measured from the test images, over a region comprising the central core of the trabecular bone [20], were identical to within experimental error at $170 \pm 15$ $\mathrm{mg} \mathrm{cm}{ }^{-3}$. The BMDs for the clinical patients, and their resulting designations compared to the mean age- and sex-matched value for a normative population of the same ethnicity [21,22], are shown in Table 1.

Table 2 shows summary statistics for the images. With the four test images the compression rate increases monotonically with slice thickness whilst the entropy and average fractal dimension decrease monotonically, as expected for a set of images that is progressively blurred. By comparison, the ten clinical images (all taken with a $5 \mathrm{~mm}$ slice thickness) have lower compression rates and higher entropy. The images from patients A and B (high BMD) and patients H, I and J (very low BMD) show the most redundancy and lowest entropy, the images from patients $\mathrm{C}, \mathrm{D}$ and $\mathrm{E}$ (normal BMD) show less redundancy and higher entropy, and the images from patients $F$ and $G$ (low BMD) show the least redundancy and highest entropy. There is a significant correlation (Pearson's correlation coefficient, $r=0.87$ ) between the effective entropy and the average fractal dimension of the ten images, although the limitations of using a global fractal dimension to characterize image texture are well documented [10,23-25]. The correlation is negative and is less significant ( $r=-0.17$ ) amongst the images $C$ to $G$, indicating that the global fractal dimension may be of little use in distinguishing images corresponding to normal and low BMDs.

Table 2

Summary statistics of the images ${ }^{\mathrm{a}}$ 


\begin{tabular}{llll}
\hline Image & $\begin{array}{l}\text { Huffinann } \\
\text { compression } \\
(\%)\end{array}$ & $\begin{array}{l}\text { Huffmann effective Average } \\
\text { entropy (bits/byte) }\end{array}$ & $\begin{array}{l}\text { fractal } \\
\text { dimension }\end{array}$ \\
\hline Test $(1 \mathrm{~mm})$ & 27.1 & 5.84 & 2.75 \\
Test $(3 \mathrm{~mm})$ & 27.2 & 5.82 & 2.72 \\
Test $(5 \mathrm{~mm})$ & 28.3 & 5.73 & 2.68 \\
Test $(10 \mathrm{~mm})$ & 29.9 & 5.61 & 2.63 \\
A & 18.5 & 6.52 & 2.50 \\
B & 12.5 & 7.02 & 2.55 \\
C & 5.4 & 7.57 & 2.69 \\
D & 4.1 & 7.68 & 2.71 \\
E & 2.6 & 7.79 & 2.74 \\
F & 1.1 & 7.81 & 2.65 \\
G & 1.4 & 7.86 & 2.68 \\
H & 8.4 & 7.33 & 2.58 \\
I & 8.3 & 7.34 & 2.57 \\
J & 8.5 & 7.31 & 2.55 \\
\hline
\end{tabular}

${ }^{\mathrm{a}}$ Each estimate for the test images is the mean from four vertebrae.

\subsection{Lacunarity of the test images}

The lacunarity plots resulting from global thresholding of a typical group of test images, identical except for acquisition slice thickness, are shown in Fig. 1. Fig. 1(a) shows the plots when the median of the pixel values was used for thresholding, Fig.1(b) when Otsu thresholding (based on discriminant analysis [17]) was used, and Fig.1(c) when the mean was used. The plots lie close to each other and have limited linearity, confirming that the images are multi-fractal and that a single fractal dimension computed for each over a specific range of window sizes would likely be of limited use in distinguishing them. Only median thresholding [Fig. 1(a)] results in plots which show lacunarity varying monotonically with slice thickness, which is the expected behaviour. For a series of progressively blurred grey-scale images, the standard deviations of the images will become monotonically smaller. After thresholding, the binarized images corresponding to the more blurred grey-scale images would be expected to show progressively less 'bone' (i.e., foreground) and consequently larger intercepts on the lacunarity plot. This trend is evident in Fig. 1(a) for median thresholding, but not in Fig. 1(b) or (c) for Otsu and mean thresholding, again indicating that median slicing is more appropriate. Similar results were obtained for the other three groups of test images (not shown). 

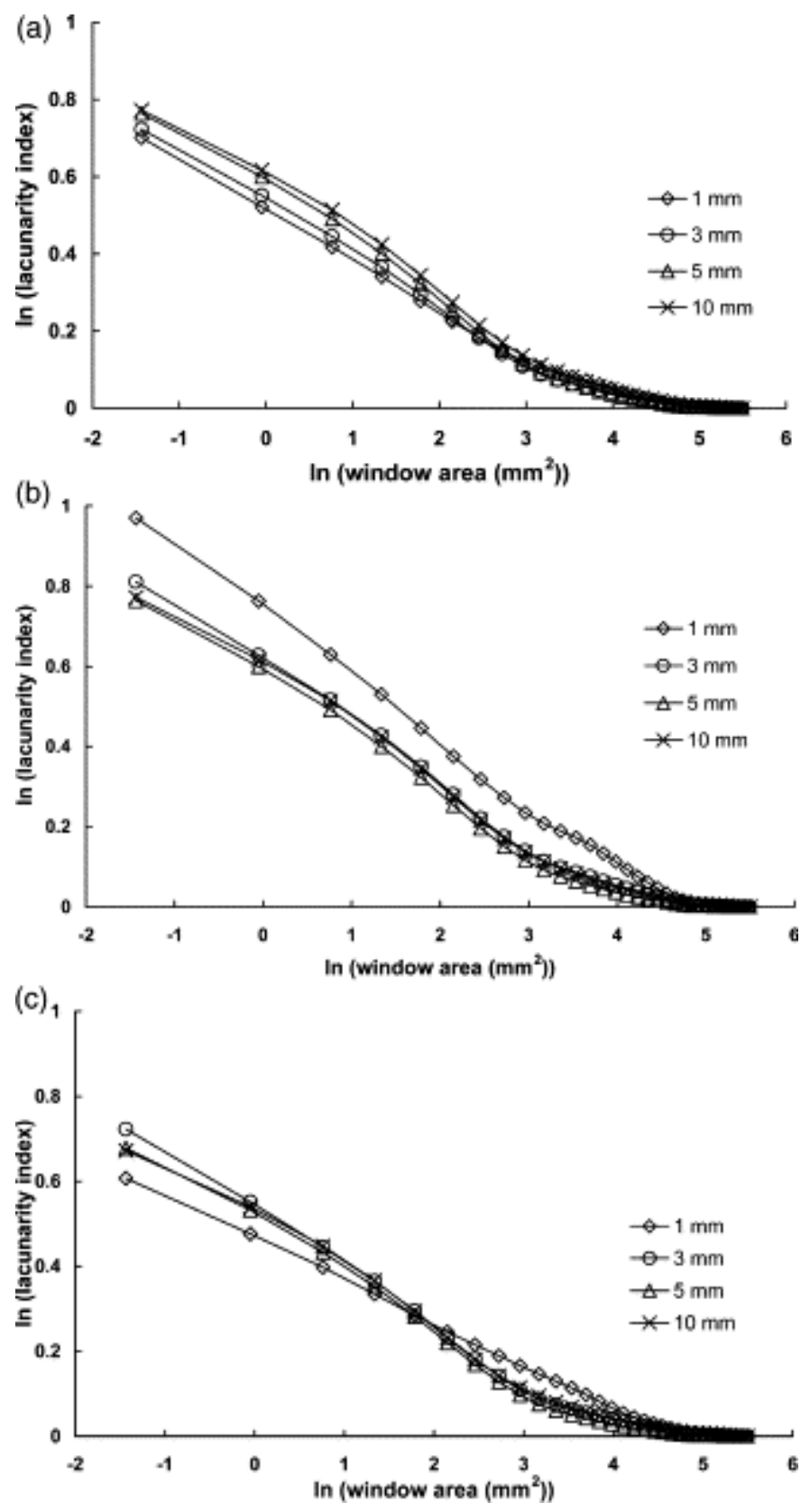

Fig. 1. Lacunarity plots for a typical group of test images, acquired at different slice thicknesses, using (a) median (b) Otsu and (c) mean thresholding.

Fig. 2(a) shows the percentage deviations from self-similarity for different window sizes, using median thresholding, for the same group of test images and demonstrates an increase in spatial homogeneity with greater slice thickness. This agrees with visual inspection of all the binarized test images: as the slice thickness increases, small 'bone' islands disappear, adjacent medium-sized areas tend to coalesce and large complex areas become smoother. The window areas corresponding to the maximum deviation are indicative of the typical size of the structuring element (e.g. marrow space). The values are more readily obtained from the zerocrossings of the first derivative of the deviation 
[Fig. 2(b)]. The average structural unit size is $18.0 \pm 4.0 \mathrm{~mm}^{2}$ in the sharpest ( $1 \mathrm{~mm}$ slice thickness) images, $19.5 \pm 4.0 \mathrm{~mm}_{2}$ in the images acquired with a $3 \mathrm{~mm}$ slice thickness, and $22.0 \pm 5.0 \mathrm{~mm}^{2}$ in the most blurred (5 and $10 \mathrm{~mm}$ slice thickness) images. The increase in apparent size is the expected effect of the reduced proportion of 'bone' with blurring. The derivative plots show second zerocrossings at window sizes of $80-100 \mathrm{~mm}_{2}$, which may indicate that there is another level of organization comprising units of four of the smaller structures. The corresponding plots for mean and Otsu thresholding (not shown) failed to show a monotonic change in aggregation with slice thickness, and consequently these schemes were not considered further.

(a)

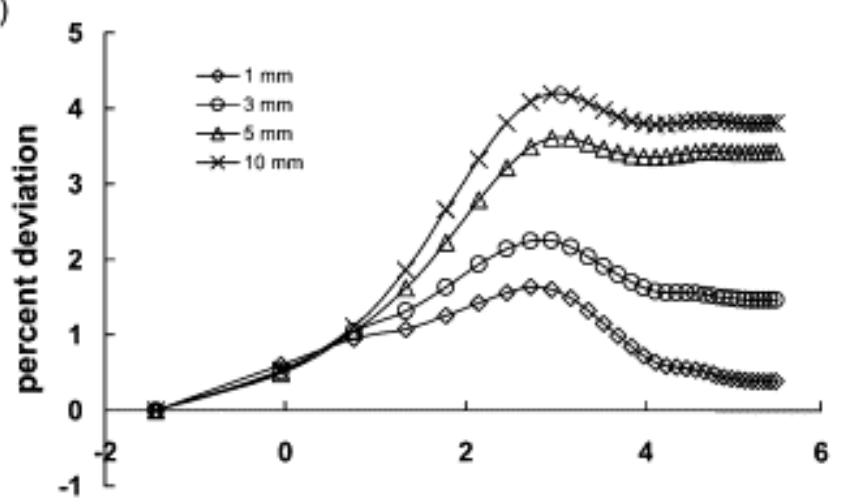

In (window area $\left.\left(\mathrm{mm}^{2}\right)\right)$

(b)

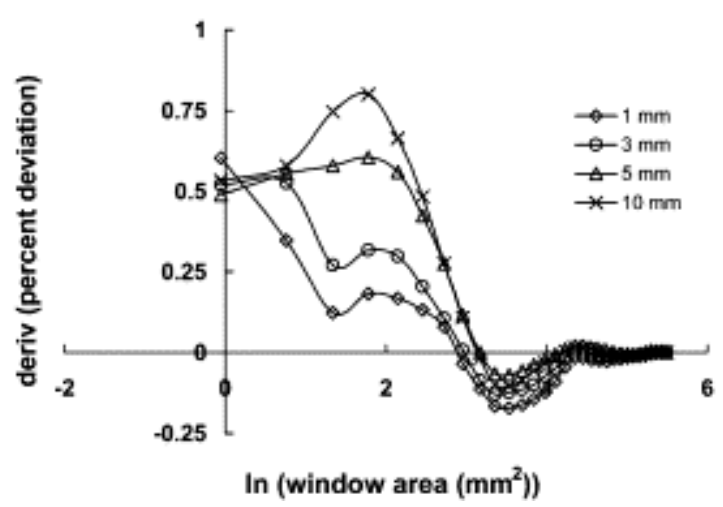

Fig. 2. (a) Percentage deviation from selfsimilarity of a typical group of test images for different window sizes, using median thresholding. (b) First derivative plot of Fig. 2(a).

Fig. 3 shows typical test images (for slice thicknesses of 1 and $10 \mathrm{~mm}$ ), and the resulting binarized images when local adaptive thresholding was implemented: each pixel was designated 'bone' or 'non-bone' depending on whether its value was greater or less than the mean of its local 7x7 neighbourhood. The resulting lacunarity plots [Fig. 4(a)] are more linear than those obtained with global thresholding, and show the expected trend of increased lacunarity with increasing slice thickness. Fig. 4(b) shows that the deviation of the locally thresholded images from self-similarity is significantly smaller than for the 
(globally) median thresholded images, but the trend with slice thickness is similar with the spatial homogeneity increasing with slice thickness. The structural unit size corresponding to the window size at which a first maximum deviation occurs can be obtained from the zero-crossing of the derivative of the deviation [Fig. 4(c)]. The average values for the 5 and $10 \mathrm{~mm}$ images (17.5 \pm 4.0 and $19.5 \pm 4.0 \mathrm{~mm}^{2}$ respectively) are close to the values obtained using median thresholding. The derivatives of the deviations for the other two images ( 1 and $3 \mathrm{~mm}$ ) just fail to cross the zero axis, due to some presumed systematic error of unknown origin. However, if the values of their closest approach to the axis are taken, the corresponding mean window size for the 1 and $3 \mathrm{~mm}$ images is $14.0 \pm 4.0 \mathrm{~mm}^{2}$, close to the values obtained using median thresholding.

(a)

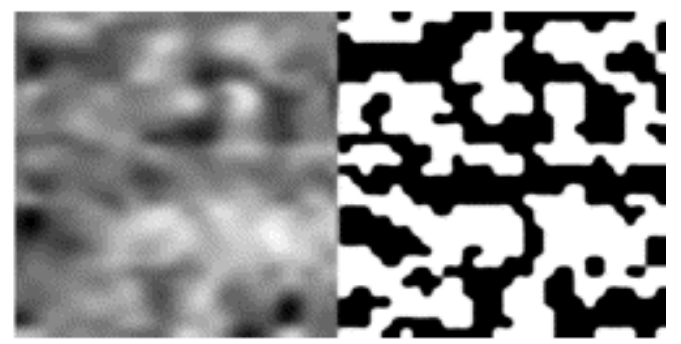

(b)

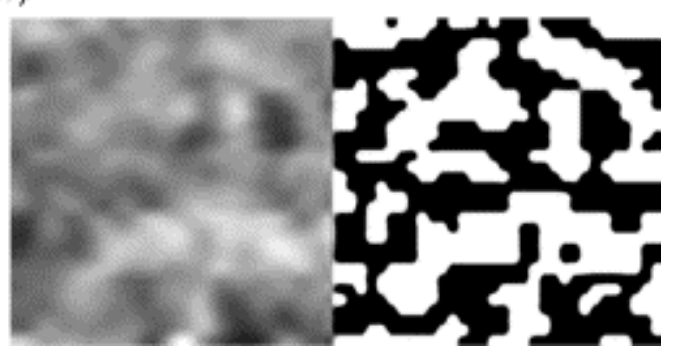

Fig. 3. (a) Typical test image (taken at $1 \mathrm{~mm}$ scan thickness) and the result of binarization using local adaptive thresholding with a $7 \cdot 7$ window. (b) Typical test image (taken at $10 \mathrm{~mm}$ scan thickness) and the result of binarization using local adaptive thresholding with a $7 \cdot 7$ window. 

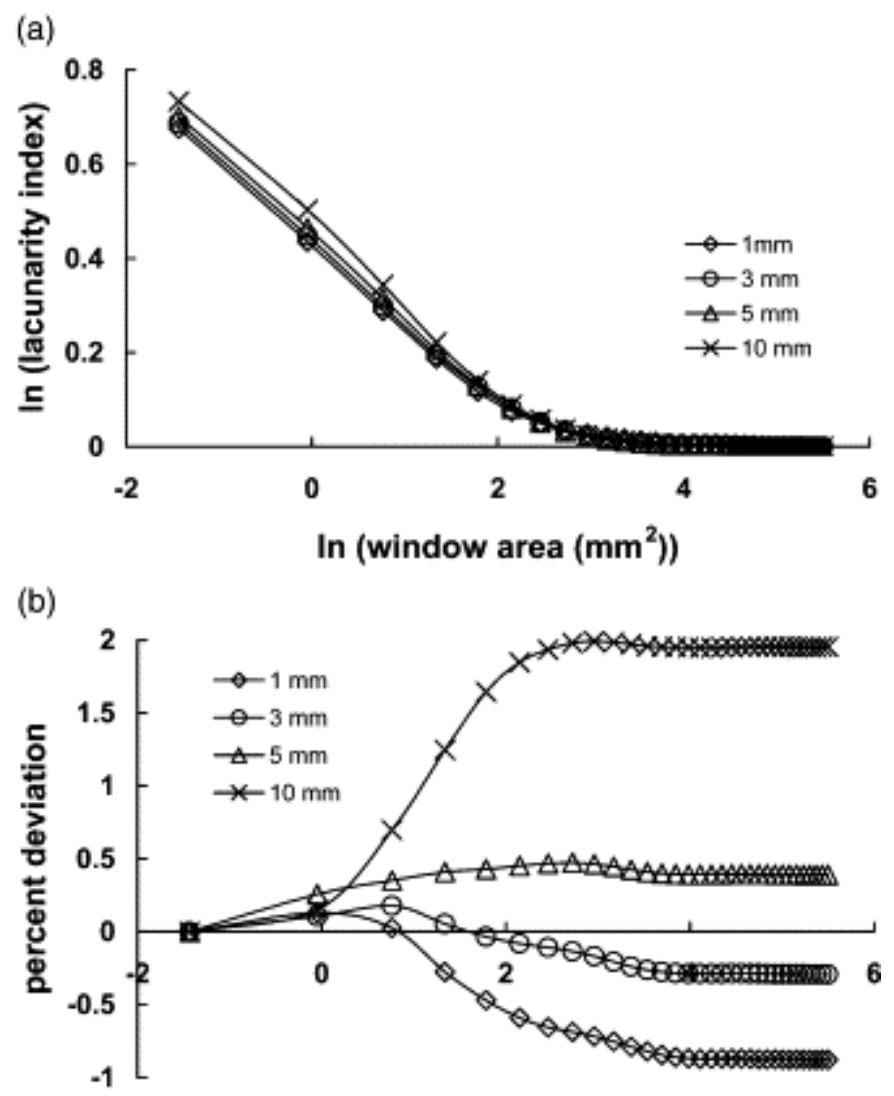

(c)

In (window area $\left.\left(\mathrm{mm}^{2}\right)\right)$

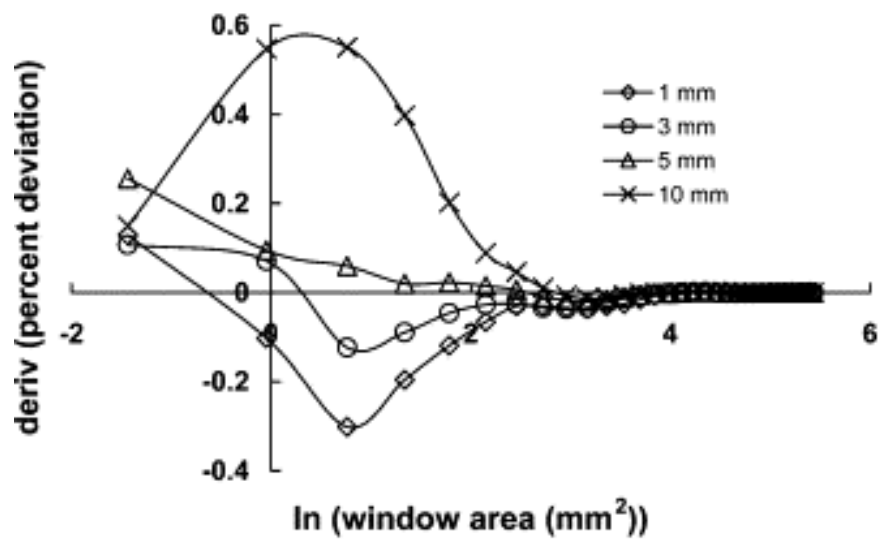

Fig. 4. (a) Lacunarity plots for a typical group of test images, acquired at different slice thicknesses, using local adaptive thresholding with a $7 \times 7$ window. (b) Percentage deviation from self-similarity of these test images for different window sizes, using local adaptive thresholding.

(c) First derivative plot of Fig. 4(b).

\subsection{Lacunarity of the clinical images}

Fig. 5 shows examples of the clinical images ( $\mathrm{A}$ and $\mathrm{F}$ ), and the results of binarization using local adaptive thresholding. The lacunarity plots for all ten clinical images are shown in Fig. 6(a). The plots are typically non-linear indicating a high degree of multifractalness: they are distinguishable from each other although no simple trend is evident. 
The deviations from self-similarity are shown in Fig. 6(b). All the images show positive deviations, indicating greater spatial homogeneity than the scale-invariant (fractal) neutral model. The deviations show a sigmoidal shape with window size, reaching characteristic maximum levels. The plots from images $\mathrm{H}$, I and $\mathrm{J}$ are very similar to each other, but noticeably different from the others because they reach their maximum levels at considerably larger window sizes. There are no maxima for any of the plots, so that the derivatives [Fig. 6(c)] do not show zero-crossings.

(a)

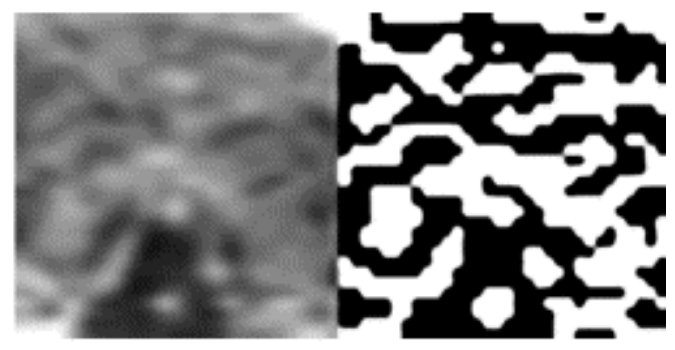

(b)

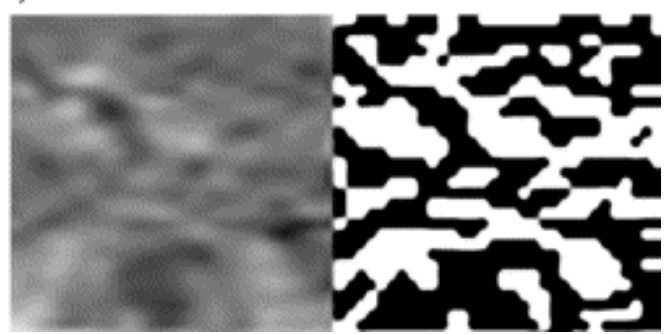

Fig. 5. (a) Image A and the result of binarization (using local adaptive thresholding and $7 \times 7$ window). (b) Image $\mathrm{F}$ and the result of binarization (using local adaptive thresholding and a $7 \times 7$ window). 
(a)

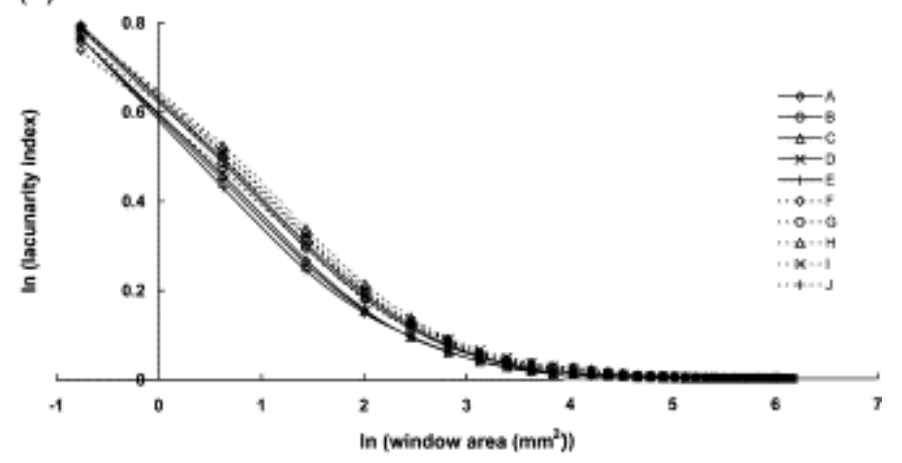

(b)
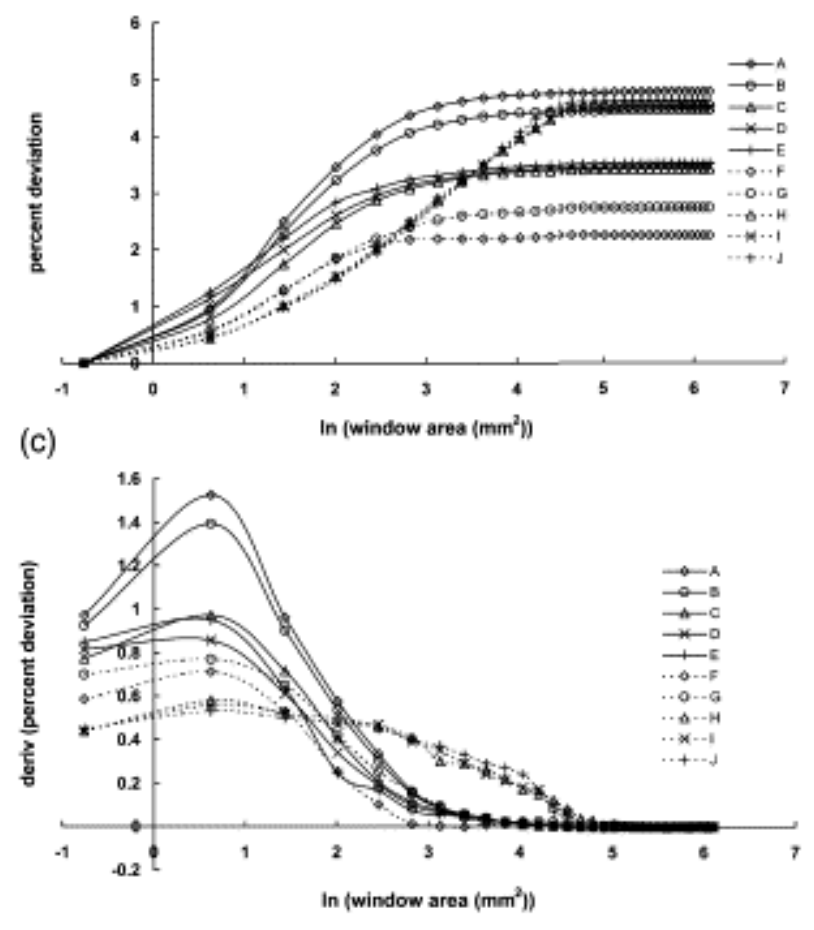

Fig. 6. (a) Lacunarity plots for the clinical images using local adaptive ( $7 \times 7$ window) thresholding. (b) Percentage deviation from self-similarity of the clinical images for different window sizes, using local adaptive thresholding.

(c) First derivative plot of Fig. 4(b).

The values of the maximum (percent) deviations (MAXDEV) from neutral (fractal) behaviour for each image are plotted against the corresponding BMDs in Fig. 7. It is difficult to assess precisely from Fig. 6(b) at what window sizes these maximum values are first achieved, so we took the window sizes (WINSIZE) at which $90 \%$ of the maximum deviation occurs to be a characteristic size. The points of inflection on the deviation graphs can be precisely identified as the maxima on the derivative graphs [Fig. 6 (c)]: we measured the value of these maxima and refer to them as MAXDERIV. The values of these derived parameters are also plotted in Fig. 7. 


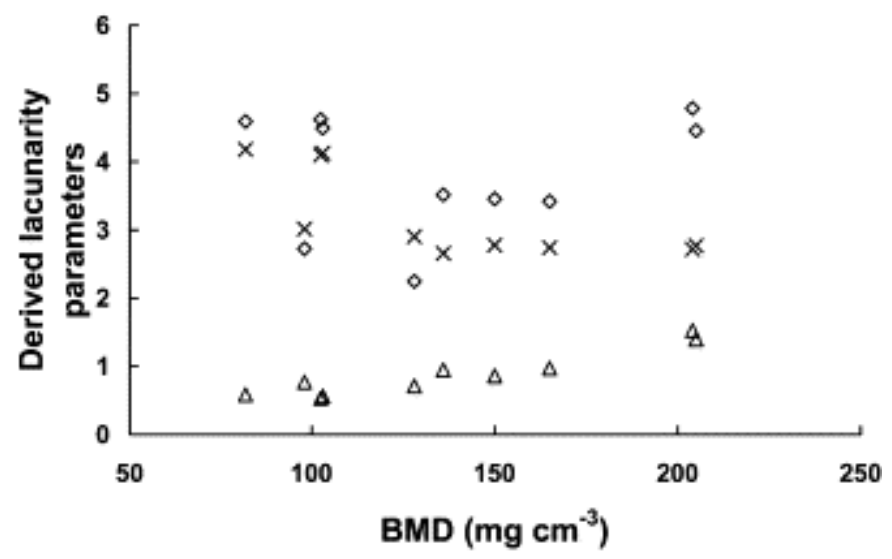

Fig. 7. The derived parameters from lacunarity analysis of the images as a function of the BMD of the patients. [MAXDEV (o) is the maximum (percent) deviation, WINSIZE $(\mathrm{x})$ is the window size (in $\mathrm{mm}^{2}$ ) at which $90 \%$ of this deviation is achieved, and MAXDERIV (+) is the maximum value of the derivative of the deviation].

\section{Discussion}

In CT images of trabecular bone the trabeculae are not properly resolved, but are blurred to sizes comparable with the scanner resolution. The observed densities at the centres of the trabeculae typically depend on local geometry and thickness and are lower than their true values, particularly for thin trabeculae. The blurring results in transition regions with observed density between bone and marrow, with the actual trabecular boundaries lying within these transition regions. Blurring is not only restricted to the plane of the CT slice $(X-Y)$, but also results from superposition in the orthogonal $(Z)$ direction as a result of finite slice thickness. The smooth range of intervening grey levels present in the original CT images is largely an artefact of the imaging system rather than real intermediate values, so that binarizing the images does not significantly reduce their actual, as opposed to apparent, information content. However, the thresholding criterion used to establish the spatial boundaries of the trabecular bone must be carefully considered, especially since the presence of such extensive blurring has degraded the grey level spectrum of the image into a single diffuse peak. The most straightforward means is by using a simple global threshold. The threshold would not be the same between different images, because of different and non-uniform bone densities between specimens and because of different acquisition conditions and differences between CT scanners. In fact, simulations of thin structures [26] have shown that no global threshold, no matter how ingeniously chosen, will produce a binarized image that presents the true thickness. Nevertheless, the use of a global threshold is so computationally attractive that it has been almost universally adopted $[6,27]$ and the judicious choice of a threshold specific to each particular image may prove to be a reasonable compromise between accuracy and computational efficiency, especially for images of high contrast. We examined four segmentation schemes in an attempt to find which could best segment blurred CT images. Using a series of test images, we found that both (global) median and local adaptive thresholding segmented the images in a consistent manner. 
The resulting estimates of marrow space size for the test images (viz., $14-18 \mathrm{~mm} 2$ for the sharpest images), derived from the deviations from lacunarity [Figs. 2(b) and 4(c)], are consistent with the trabecular separations ranging from about $2.6 \mathrm{~mm}$ for normal premenopausal women to nearly $6 \mathrm{~mm}$ for osteoporotic women determined from image analysis of high-resolution CT images of vertebral bone [28].

Osteoporosis is routinely assessed from comparison of the medullary BMD with agematched controls (Table 1). Classification can be assisted by considering the morphological pattern of trabecular bone [29], despite its degradation in the CT image: and the density profile across the trabecular region [30] leads to an analogous classification (Table 3). The fractal dimension of the density profile has been used [30] to quantitate the rather subjective visual appearance of the density profile, but we consider that the average fractal dimension of the whole spongiosa area may be more reliable. There are concerns about using fractal dimension alone to characterize pattern $[10,23]$. Furthermore, trabecular bone images are known to be multifractal [24], and the concept of a fractal signature $[10,25]$ has been invoked to characterize such textures. Notwithstanding these concerns, it is interesting that the fractal dimensions determined from the spongiosa area of our patients (Table 2) match the visual classification scheme enunciated in Table 3 with images A and B (from high BMD patients) and $\mathrm{H}$, I and $\mathrm{J}$ (from very low BMD patients) having a low fractal dimension compared with images D and $\mathrm{E}$ (from normal BMD patients) and F and G (from low BMD patients). The value of the fractal dimension does not change monotonically with BMD if healthy patients with a higher than normal BMD are included. This seems to be the cause of a reported contradiction [31]. If Type 1 (healthy) patients were excluded from our sample, the fractal dimension would appear to decrease monotonically with reducing BMD as expected intuitively with a progressive loss of trabeculae. Indeed an earlier study of dental radiographs of the maxillary anterior alveolar region [31], which contains a relatively high concentration of trabeculae, reported a monotonic decrease in fractal dimension with decalcification (viz. simulated osteoporosis). However, if Type 4 (severely osteoporotic) patients were excluded from our sample instead, it could appear that fractal dimension increases with reducing BMD, although the poor discrimination between patients with normal and low BMD is a confounding factor. Such a trend has been reported in studies of radiographic images of a human calcaneous (heel) bone during immobilization [32] and of mandibular alveolar bone after decalcification [33]. Clearly, changes in fractal dimension need to be interpreted with care. Table 3 suggests that both healthy and severely osteoporotic bone will generally have a low fractal dimension (with the latter being multi-fractal), and normal and osteopenic bone will have higher fractal dimension and will be difficult to distinguish on the basis of fractal dimension alone.

Table 3. A visual classification scheme for the assessment of the trabecular structure as used for the determination of the degree of osteoporosis (columns 1, 3 and 5 are after Ref. [30]: columns 2, 4 and 6 were added inferentially by the authors) 


\begin{tabular}{|c|c|c|c|c|c|}
\hline Type & BMD & Spongiosa pattem & Marrow size & Density profile & $\begin{array}{l}\text { Fractal dimension of } \\
\text { ROI }\end{array}$ \\
\hline 1: Healthy & High & $\begin{array}{l}\text { Homogeneously dense with } \\
\text { granular stucture }\end{array}$ & $\begin{array}{l}\text { Small, } \\
\text { homogeneous }\end{array}$ & $\begin{array}{l}\text { Homogeneous at high } \\
\text { sponsiosa density }\end{array}$ & Low, fractal \\
\hline $\begin{array}{l}\text { 2: Beginning } \\
\text { demineralization }\end{array}$ & Normal & $\begin{array}{l}\text { Discrete disseminated } \\
\text { intertrabecular areas }\end{array}$ & $\begin{array}{l}\text { Medium, } \\
\text { inhomogeneous }\end{array}$ & $\begin{array}{l}\text { Inhomogeneous at high } \\
\text { spongiosa density }\end{array}$ & High, multi-fiactal \\
\hline 3: Osteopenia & Low & $\begin{array}{l}\text { Confluent intertrabecular areas less } \\
\text { than } 50 \% \text { of the cross-sectional } \\
\text { surface }\end{array}$ & $\begin{array}{l}\text { Large, } \\
\text { inhomogeneous }\end{array}$ & $\begin{array}{l}\text { Inhomogeneous at low } \\
\text { spongiosa density }\end{array}$ & High, multi-fractal \\
\hline 4: Severe osteoporosis & Very low & $\begin{array}{l}\text { Confluent intertrabecular areas } \\
\text { more than } 50 \% \text { of the cross- } \\
\text { sectional surface }\end{array}$ & $\begin{array}{l}\text { Very large, } \\
\text { homogeneous }\end{array}$ & $\begin{array}{l}\text { Mostly homogeneous at } \\
\text { low sponsiosa density }\end{array}$ & Low, multi-fractal \\
\hline
\end{tabular}

The trend in the maximum (percent) deviations (MAXDEV) from fractalness (i.e. degree of homogeneity) for the clinical images is interesting (Fig. 7). The images from patients $\mathrm{A}$ and $\mathrm{B}$ (high BMD) show the most homogeneity (i.e. largest maximum deviation): images $\mathrm{C}, \mathrm{D}$ and $\mathrm{E}$ (normal BMD) are less homogeneous, and images $\mathrm{F}$ and $\mathrm{G}$ (low BMD) are less homogeneous still: whilst images H, I and J (very low BMD) have a homogeneous structure similar to images A and B. This relationship between degree of homogeneity and BMD is consistent with the visual classification of Table 3.

The absence of maxima in the deviation plots for the clinical images [Fig. 6(b)] was thought to be related to the use of $5 \mathrm{~mm}$ scan thicknesses, a consequence of our desire to limit patient exposure, and the consequent blurring: however, two patients (B and F) were re-scanned using a $1 \mathrm{~mm}$ scan thickness, and the subsequent deviation plots showed similar sigmoidal shapes with no maxima (not shown). The shape of the deviation plots are the result of a complex distribution of marrow space sizes, and confounded our attempts to define a characteristic marrow space size for each image. Nevertheless, the shapes of these plots presumably contain information on the underlying distributions: and they can be characterized by the window sizes where the plots first reach their maximum values and by the curvature of the sigmoidal plots. Both these measures are difficult to determine precisely, but are related to the window sizes at which $90 \%$ of the maximum values occur (WINSIZE) and the maximum value of the derivatives of the percentage deviation (MAXDERIV) respectively. These measures are shown in Fig. 7. WINSIZE is a measure of the characteristic marrow space size: it increases slightly through images A$\mathrm{G}$ (viz. those assessed as having high, normal or low BMDs) but jumps significantly for images $\mathrm{H}$, I and J (viz. those from pataients with very low BMDs). MAXDERIV is another derived parameter related to marrow space size. It is strongly correlated with BMD ( $r$ 0.939), the positive correlation with BMD indicating that it is inversely related to marrow space size. Comparison of the values obtained for WINSIZE and MAXDERIV with 'gold standard' values, measured histologically [34], for a larger sample of patients would be needed in order to establish how valid they are as indicators of marrow space size and which of them is the more sensitive.

During ageing and osteoporosis, the trabecular network in human vertebrae loses connectivity through a thinning and loss of trabeculae accompanied by an increased spacing between them [35,36] with a consequent loss of bone strength and increased fracture risk [37]. Numerous studies have attempted to use histomorphometric indices such as trabecular thickness (Tb.Th) and trabecular separation (Tb.Sp) [38,39], or extensions of these to digitized projectional overlays [40,41], to follow these structural changes. However these indices are first order statistical measures similar to the standard 
deviation of the grey level values of thresholded images: it has been recognized that second order statistical measures which quantify the relationship between neighbouring pixels would likely provide more information on the pattern of trabeculation [41]. Threedimensional histomorphometric indices have also been invoked [6]. Unfortunately, these indices assumed a plate model [38] for the trabecular bone in their derivations. Although the parallel plate model accurately describes the trabecular bone in the femoral head, it is not appropriate for the lumbar spine in which the trabecular bone has a significant rodlike component $[6,42]$. Our study suggests that lacunarity, which is a second order statistical measure, can successfully characterize the trabeculation pattern in vertebral bone with sufficient sensitivity to distinguish different degrees of bone quality.

Even though the images are poorly resolved, the resulting estimates of lacunarity and fractal dimension from appropriately thresholded CT images of the lumbar vertebrae are not significantly different from those obtained with high-resolution images [9]. As shown histologically, the existence of marrow spaces in the trabecular network and their size determines the structural competence of the bone network [34]. As osteoporosis proceeds, and trabeculae are thinned and lost, the remaining trabeculae are more widely separated, less connected, and less likely to withstand a compressive force [35]. Such a pattern of bone loss can best be followed by examining the homogeneity of the marrow spaces, which is related to the connectivity of the bone network, and can be determined from the lacunarity of the image. The marrow space size in thresholded CT [43] and magnetic resonance [44] images has been investigated using a region growing algorithm, and compared with a putative connectivity index. The marrow space increased with bone loss, and the connectivity index decreased. However a wide variation in marrow space sizes was found within each subject: although the majority of spaces were less than 0.5 $\mathrm{mm} 2$, the presence of a few large holes resulting from breaks in the network skews the average values. This wide distribution in marrow space sizes may be the reason why a mean size could not be determined from our deviation plots derived from lacunarity. Notwithstanding, it is clear that the values of maximum deviation (and the maximum derivatives of the deviation) incorporate this distribution and reflect bone connectivity and hence bone strength.

Our preliminary results on a limited patient sample indicate that, provided local adaptive thresholding is adopted, derived parameters (such as the maximum derivative of the deviation (MAXDERIV) and the window size at 90\% maximum deviation (WINSIZE), and the maximum deviation from fractality (MAXDEV)) can successfully distinguish different degrees of osteoporosis through their ability to monitor marrow spaces and their connectivity. Even small ROIs (e.g. 32x32) in a vertebral bone image can be used. Lacunarity analysis of the vertebral trabecular texture is a promising additional diagnostic tool to complement BMD in assessing bone quality to characterize osteoporosis and to improve the prediction of individual fracture risk. We are currently applying the technique to a much larger group of patients in order to establish its diagnostic value. Given the standard deviation of the variables in this pilot study, at least 30 patients would be required to demonstrate an association between the image parameters and the BMD with a statistical power of 0.8 at a probability level, $p$, of 0.05 .

\section{Acknowledgements}




\section{This study was supported by Kuwait University.}

\section{References}

[1] Kleerekoper M, Villaneuva AR, Stanciu J, Rao DS, Parfitt AM. The role of three dimensional trabecular microstructure in the pathogenesis of vertebral compression fractures. Calcif Tissue Int 1985;37:594-7.

[2] Goldstein SA, Goulet R, McCubbrey D. Measurement and significance of three-dimensional architecture to the mechanical integrity of trabecular bone. Calcif Tissue Int 1993;53(1):5127-33.

[3] Mu“ller R, Hildebrand T, Ru“egsegger P. Non-invasive bone biopsy: a new method to analyse and display the three-dimensional structure of trabecular bone. Phys Med Biol 1994;39:145-64.

[4] Mu“ller R, Hildebrand T, Ha“uselmann HJ, Ru“egsegger P. In vivo reproducibility of three-dimensional structural properties of noninvasive bone biopsy using 3D-pQCT. J Bone Mineral Res 1996;11:1745-50.

[5] Peyrin F, Houssard JP, Maurincomme EM, Peix G, Goutte R, Laval-Jeantet AM, Amiel M. 3D display of high resolution vertebral structure images. Comp Med Imag Graphics 1993;17:251-6.

[6] Mu“ller R, Van Campenhout H, Van Damme B, Van der Perre G, Dequeker J, Hildebrand T, $\mathrm{Ru}$ “egsegger P. Morphometric analysis of human bone biopsies: a quantitative structural comparison of histological sections and micro-computed tomography. Bone 1998;23:59-66.

[7] Weszka JS, Dyer CR, Rosenfeld A. A comparative study of texture measures for terrain classification. IEEE Trans Systems Man and Cybernetics 1976;6:269-85.

[8] Haralick RM. Statistical and structural approaches to texture. Proc 4th Int Joint Conf Pattern Recog 1979:45-60.

[9] Dougherty G. A comparison of the texture of computed tomography and projection radiography images of vertebral trabecular bone using fractal signature and lacunarity. Med Eng Phys 2001;23:313-21.

[10] Dougherty G, Henebry G. Fractal signature and lacunarity in the measurement of the texture of trabecular bone in clinical CT images. Med Eng Phys 2001;23:369-80.

[11] Mandelbrot BB. The fractal geometry of nature. New York: Freeman, 1983.

[12] Allain C, Cloitre M. Characterizing the lacunarity of random and deterministic fractal sets. Physical Review Letters 1991;A44:3552-8.

[13] Plotnick RE, Gardner RH, O’Neill RV. Lacunarity indices as measures of landscape texture. Landscape Ecology 1993;8:201-11.

[14] Weishampel JF, Sloan JH, Boutet JC, Godin JR. Mesoscale changes in textural pattern of 'intact' Peruvian rainforests (1970s-1980s). Int J Remote Sensing 1998;19:1007-14.

[15] Henebry GM, Kux HJH. Lacunarity as a texture measure for SAR imagery. Int J Remote Sensing 1995;16:565-71.

[16] Sahoo PK, Soltani S, Wong AK. A survey of thresholding techniques. Comput Vision Graphics Image Process 1988;41:233-60. 
[17] Otsu N. Threshold selection method from grey-level histograms. IEEE Trans Systems Man Cyber 1979;9:62-6.

[18] Ouyang X, Majumdar S, Link TM, Lu Y, Augat P, Lin J, Newitt D, Genant HK. Morphometric texture analysis of spinal trabecular bone structure assessed using orthogonal radiographic projections. Med Phys 1998;25:2037-45.

[19] Pentland AP. Fractal-based description of natural scenes. IEEE Trans Pattern Anal Machine Intell 1984;6:661-74.

[20] Dougherty G. Quantitative CT in the measurement of bone quantity and bone quality for assessing osteoporosis. Med Eng Phy 1996;18:557-68.

[21] Kalender WA, Felsenberg D, Louis O, Lopez P, Klotz E, Osteaux M, Fraga J. Reference values for trabecular and cortical bone density in single and dual-energy quantitative computed tomography. Eur $\mathrm{J}$ Radiol 1989;9:75-80.

[22] Dougherty G, Al-Marzouk N. Bone density measured by dual-energy X-ray absorptiometry in healthy Kuwaiti women. Calcif Tissue Int 2001;68:225-9.

[23] Garding J. Properties of fractal intensity surfaces. Pattern Recog Lett 1988;8:319-24.

[24] Buckland-Wright JC, Lynch JA, Rymer J, Fogelman I. Fractal signal analysis of macroradiographs measures trabecular organization in lumbar vertebrae of postmenopausal women. Calcif Tissue Int 1994;54:106-12.

[25] Lynch JA, Hawkes DJ, Buckland-Wright JC. Analysis of texture in macroradiographs of osteoarthritic knees using the fractal signature. Phys Med Biol 1991;36:709-22.

[26] Dougherty G, Newman D. The measurement of thickness and density of thin structures by CT: a simulation study. Med Phys 1999;26:1341-8.

[27] Geraets WGM, Van der Stelt PF. Analysis of the radiographic trabecular pattern. Patt Recog Lett 1991;12:575-81.

[28] Mundinger A, Wiesmeier B, Dinkel E, Helwig A, Beck A, Moenting JS. Quantitative image analysis of vertebral body architecture- improved diagnosis in osteoporosis based on high-resolution computed tomography. Brit J Radiol 1993;66:209-13.

[29] Andresen R, Radmer S, Hakim S, Banzer D, Stolpmann HJ. Optimizing QCT diagnosis of osteoporosis by including a morphological analysis of vertebral spongiosa. Int J Bone Miner Calcium Regulation Res 1994;25(2 (Suppl. 2)):14.

[30] Haidekker MA, Andresen R, Evertsz CJG, Banzer D, Pietgen HO. Assessing the degree of osteoporosis in the axial skeleton using the dependence of the fractal dimension on the grey level threshold. Brit J Radiol 1997;70:586-93.

[31] Southard TE, Southard KA. Detection of simulated osteoporosis in maxillae using radiographic texture analysis. IEEE Trans Biomed Eng 1996;43:123-32.

[32] Lundahl T, Ohley WJ, Kay SM, Siffert R. Fractional Browman motion: a maximum likelihood estimator and its application to image texture. IEEE Trans Med Ima 1986;5:152-61.

[33] Ruttimann UE, Weber RL, Hazelrig JB. Fractal dimension from radiographs of peridental alveolar bone: a possible diagnostic indicator of osteoporosis. Oral Surg Oral Med Oral Pathol 1992;74:98-110. 
[34] Vesterby A, Gundersen HJG, Melsen F. Star volume of marrow space and trabeculae of the first lumbar vertebra: sampling efficiency and biological variation. Bone 1989;10:7-13.

[35] Weinstein RS, Hutson MS. Decreased trabecular width and increased trabecular spacing contribute to bone loss with ageing. Bone 1987;8:137-42.

[36] Bergot C, Laval-Jeantet AM, Preteux F, Meunier A. Measurement of anisotropic vertebral trabecular bone loss during ageing by quantitative image analysis. Calcif Tissue Int 1988;43:143-9.

[37] Mosekilde L, Danielsen CC. Biomechanical competence of vertebral trabecular bone in relation to ash density and age in normal individuals. Bone 1987;8:79-85.

[38] Parfitt AM, Mathews CHE, Villeneuva AR, Kleerekoper M, Frame B, Rao DS. Relationship between surface volume and thickness of iliac trabecular bone in ageing and in osteoporosis: implications for the microanatomic and cellular mechanism of bone loss. J Clin Invest 1983;72:1396-409.

[39] Feldkamp LA, Goldstein SA, Parfitt AM, Jesion G, Kleerekoper M. The direct examination of threedimensional bone architecture in vitro by computed tomography. J Bone Min Res 1989;4:3-11.

[40] Durand EP, Ru"egsegger P. Cancellous bone structure: analysis of high-resolution CT images with the run-length method. J Comp Assist Tomog 1991;15:133-9.

[41] Ouyang X, Majumdar S, Link TM, Lu Y, Augat P, Lin J, Newett D, Genant HK. Morphometric texture analysis of spinal trabecular bone structure assessed using orthogonal radiographic projections. Med Phys 1998;25:2037-45.

[42] Day JS, Ding M, Odgaard A, Summer DR, Hvid I. Weinans Parallel plate model for trabecular bone exhibits volume fractiondependent bias. Bone 2000;27:715-20.

[43] Gordon CL, Webber CE, Adachi JD, Christoforou N. In vivo assessment of trabecular bone structure at the distal radius from high-resolution computed tomography images. Phys Med Biol 1996;41:495-508.

[44] Gordon CL, Webber CE, Christoforou N, Nahmias C. In vivo assessment of trabecular bone structure at the distal radius from high-resolution magnetic resonance images. Med Phys 1997;24:585-93. 